\title{
Pentafluorophenyl vinyl sulfonate enables efficient, metal-free, radical- based alkene hydroacylation with aldehyde as limiting reagent
}

\author{
Vijay Chudasama* \\ Received (in $X X X, X X X)$ Xth $X X X X X X X X X 20 X X$, Accepted $X$ th $X X X X X X X X X 20 X X$ \\ ${ }_{5}$ DOI: $10.1039 /$ b000000x
}

The unique properties of pentafluorophenyl vinyl sulfonate allow for a hitherto unmet feat to be realised; efficient and high yielding, metal-free, radical-based alkene hydroacylation employing aldehyde as limiting reagent. The optimised 10 conditions are shown to work in good yields across a series of aldehydes, thus demonstrating the wide applicability of the developed protocol.

Free radical based methodologies offer a complementary approach to the conditions often required for two electron 15 processes, as exemplified in the important role they have played in synthetic organic chemistry. ${ }^{1}$ The first application of free radical chemistry in the hydroacylation field came in 1949, where Kharasch reported the formation of unsymmetrical ketones from reaction of aldehydes with alkenes using diacetyl peroxide as a 20 thermally activated initiator. ${ }^{2}$ Soon after the work by Kharasch, Patrick and Huang reported the benzoyl peroxide-initiated addition of aldehydes to numerous electron deficient alkenes. ${ }^{3}$ Numerous other examples based on free radical chemistry have been reported since these early articles, with strained double 25 bonds and perfluoroalkenes proving to be particularly good acyl radical acceptors. ${ }^{4}$ However, an important trend from the early reports to date is the use of the aldehyde component in vast excess, despite the emergence of polarity reversal catalysts. ${ }^{4 \mathrm{~g}, 5}$ Although, transition metal-based hydroacylation protocols have 30 achieved hydroacylation using aldehyde as limiting reagent, these protocols come with the disadvantages associated with such procedures (e.g. cost, limited aldehyde scope, toxicity, appreciable decarbonylation). ${ }^{4 \mathrm{~g}, 6}$ Advances in base promoted $\mathrm{N}$-heterocyclic carbene-catalysed intermolecular hydroacylation 35 have also been made, but these remain almost exclusively limited to aromatic aldehydes, due to competing aldol-type chemistry, and highly strained $\pi$-acceptors (e.g. cyclopropenes and arynes). ${ }^{7}$ The lack of a free radical, transition metal-free protocol based on aldehyde functionalisation (i.e. using aldehyde as limiting 40 reagent) may be due to the alkene component not being effective enough in the hydroacylation radical pathway, i.e. an inefficient acyl radical trap and/or a poor chain propagator.

Recently, an aerobically-initiated hydroacylation protocol for the hydroacylation of various electron-poor alkenes (e.g. vinyl 45 sulfonates, vinyl sulfones, unsaturated esters and vinyl phosphonates) has been described. ${ }^{8}$ In this programme, pentafluorophenyl vinyl sulfonate (PFPVS) was discovered to be exceptionally well-suited to undergo efficient hydroacylation. ${ }^{8 \mathrm{f}}$
Encouraged by the favourable properties of PFPVS as a radical 50 acceptor/chain carrier, a study to develop a protocol for its use in efficient, metal-free hydroacylation with aldehyde being employed as the limiting reagent was embarked upon (see Figure 1). Owing to previous work which has shown the products of the hydroacylation of PFPVS, i.e. $\gamma$-keto-PFP-sulfonates, to undergo: 55 (i) quantitative elimination to form enones; (ii) eliminationaddition to provide an indirect route to the hydroacylation of electron rich alkenes; and (iii) facile conversion to $\gamma$-ketosulfonamides, sultams and sultones, ${ }^{8 \mathrm{~b}, 8 \mathrm{f}}$ such a protocol could find application in various fields. ${ }^{8 \mathrm{f}}$

Conventional metal-free alkene hydroacylation (alkene functionalisation)

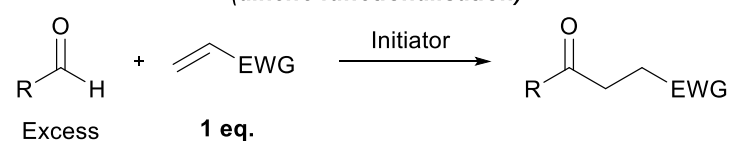

This work (aldehyde functionalisation)

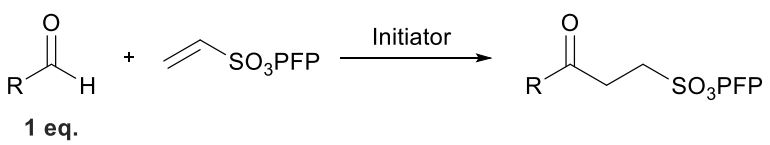

PFPVS allows for a new approach to metal-free alkene hydroacylation: From functionalising alkenes to functionalising aldehydes

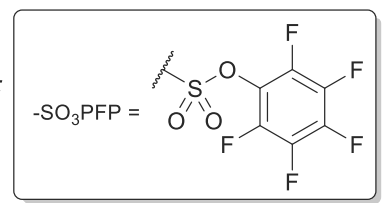

60

Fig. 1 Enabling metal-free hydroacylation with aldehyde functionalisation as its focus.

The study began by analysing the reaction of PFPVS with a model aldehyde under previously reported aerobically-initiated 65 hydroacylation conditions (Table 1, Entry 1). ${ }^{8 \mathrm{~b}}$ Aware of the well-documented issue of undesirable competing decarbonylation of secondary aldehydes in hydroacylation processes, 2-methylbutanal 1a was chosen as the model aldehyde to tackle this issue, concurrently, during optimisation. Reaction of an equivalent of 70 2-methyl-butanal 1a with PFPVS 2 under aerobic activation conditions resulted in a low yield of $\gamma$-keto-PFP-sulfonate $\mathbf{3 a}$ (Table 1, Entry 1). This was mainly due to the low conversion of alkene, which was a consequence of competing aldehyde autooxidation to acid 4. As this competing side-reaction is always 75 likely to exist in any aerobic-based hydroacylation protocol, the use of other, non-aerobic-based initiators was appraised. With the 
issue of competing decarbonylation being exacerbated with high temperature, the use of low temperature thermal initiators, lauroyl peroxide and azobisisobutyronitrile (AIBN), at $20 \mathrm{~mol} \%$, was explored. Initially, reaction of 2-methylbutanal 1a with vinyl 5 sulfonate 2 at $60{ }^{\circ} \mathrm{C}$ in a 1:1 molar ratio with both initiators was trialled (Table 1, Entries 2 and 3).

Table 1 Optimisation of reaction of 2-methylbutanal 1a with 2.

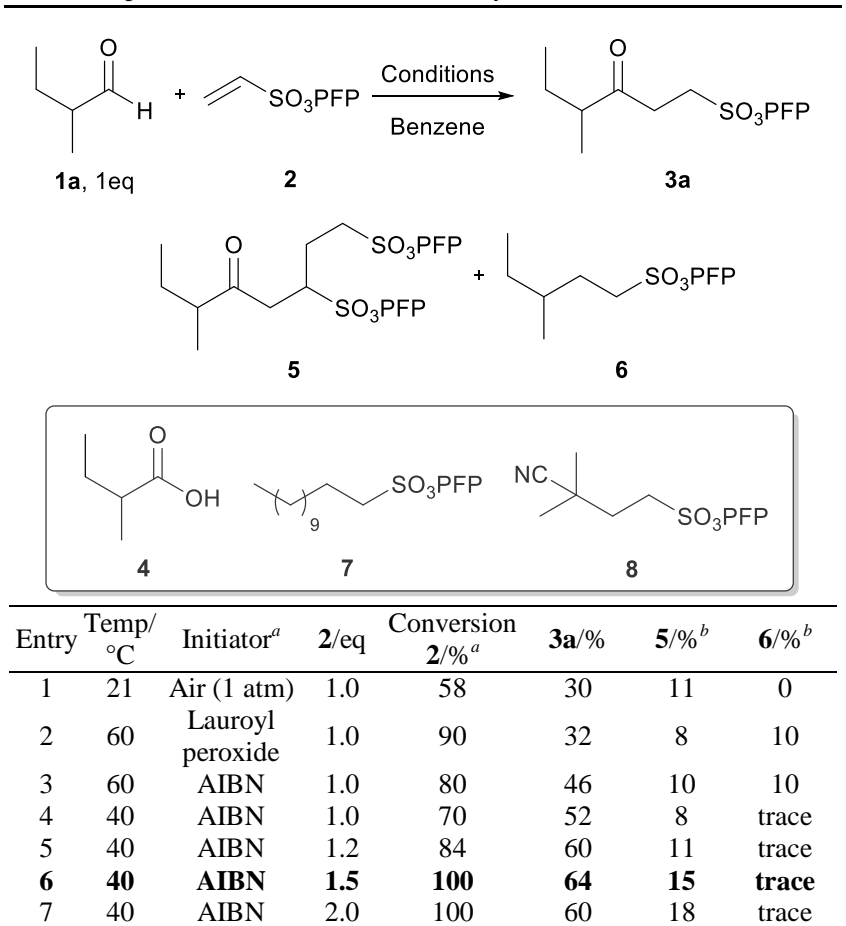

${ }^{a} 20 \mathrm{~mol} \%$ unless stated otherwise. ${ }^{b}$ Determined by integration of ${ }^{1} \mathrm{H}$ NMR relative to pentachlorobenzene as an internal standard.

10 On using lauroyl peroxide, the reaction proceeded with almost complete conversion of vinyl sulfonate, however, only a $32 \%$ yield of ketone 3a was obtained. The low yield of ketone and complete conversion of vinyl sulfonate $\mathbf{2}$ was due to the formation of a significant amount of alkyl sulfonate 7; 15 presumably derived from undecyl radical (generated from thermal decomposition of lauroyl peroxide) addition to vinyl sulfonate 2 . In the case of AIBN, reaction at $60{ }^{\circ} \mathrm{C}$ with a $1: 1$ molar ratio of 1a:2 resulted in a modest yield of ketone 3a, 46\%, with the formation of double addition product $\mathbf{5}$ and decarbonylated 20 addition product 6 also being observed (Table 1, Entry 3). In contrast to lauroyl peroxide, only a trace quantity of initiator-derived alkyl sulfonate, i.e. compound $\mathbf{8}$, was observed. This may be a consequence of a less nucleophilic and more sterically crowded tertiary radical resulting from thermal 25 decomposition of AIBN. Alkyl sulfonate $\mathbf{6}$ is likely to be derived from the secondary radical formed upon decarbonylation of the acyl radical of aldehyde 1a. To reduce the formation of alkyl sulfonate $\mathbf{6}$, the effect of lowering temperature to suppress decarbonylation was explored. Gratifyingly, lowering the 30 temperature to $40{ }^{\circ} \mathrm{C}$ suppressed formation of alkyl sulfonate 6 and increased the yield of ketone $\mathbf{3 a}$ to $52 \%$ (Table 1, Entry 4). In an attempt to increase conversion of vinyl sulfonate 2 , the effect of altering the 1a:2 ratio from 1:1 to 1:2 was explored (Table 1, Entries 4-7). Despite there being an increase in the formation of
35 double addition product $\mathbf{5}$, complete conversion was achieved at 1:1.5 and 1:2 molar ratios of 1a:2. The increased conversion was attributed to more efficient acyl radical trapping by vinyl sulfonate 2 due to its higher concentration. Unsurprisingly, this also resulted in a higher yield of double addition product $\mathbf{5}$ as the 40 adduct radical that results from acyl radical addition to vinyl sulfonate $\mathbf{2}$ is also more likely to be trapped by another vinyl sulfonate. Optimal yield, 64\%, was achieved at a 1:1.5 molar ratio of 1a:2.

With optimised conditions in-hand, translation to other 45 electron deficient alkene systems was appraised. Thus the use of ethyl vinyl sulfone, an unsaturated ester and dimethyl vinyl phosphonate as the alkene component was trialled. In contrast to PFPVS 2, trace amounts of hydroacylation products were observed across the series of alkenes (Table 2, Entries 1-3). Even 50 the use of 2,4,6-trichlorophenyl vinyl sulfonate, gave a poor yield of ketone, $21 \%$ (Table 2, Entry 4). These results only serve to highlight the unique, favourable properties of PFPVS as an acyl radical acceptor/radical chain carrier.

Table 2 Reaction of a series of alkenes with 2-methylbutanal 1a.

\begin{tabular}{|c|c|c|c|c|c|}
\hline & $1 \mathrm{a}$ & $\sum_{\mathrm{R}^{1}}^{\mathrm{R}^{3}} \mathrm{R}^{2}$ & $\begin{array}{l}\mathrm{O} \mathrm{mol} \% \\
\mathrm{BN}, 40^{\circ} \mathrm{C}\end{array}$ & $\mathrm{R}^{1}$ & \\
\hline Entry & $\mathrm{R}^{1}$ & $\mathrm{R}^{2}$ & $\mathrm{R}^{3}$ & $\begin{array}{c}\text { Conversion } \\
\text { alkene } / \%^{a}\end{array}$ & $\begin{array}{c}\text { Yield of } \\
\text { Ketone } / \%\end{array}$ \\
\hline 1 & $\mathrm{H}$ & $\mathrm{SO}_{2} \mathrm{Et}$ & $\mathrm{H}$ & 12 & trace \\
\hline 2 & $\mathrm{Me}$ & $\mathrm{CO}_{2} \mathrm{Me}$ & $\mathrm{CO}_{2} \mathrm{Me}$ & 8 & trace \\
\hline 3 & $\mathrm{H}$ & $\mathrm{P}(\mathrm{O})(\mathrm{OMe})_{2}$ & $\mathrm{H}$ & 11 & trace \\
\hline 4 & $\mathrm{H}$ & $\mathrm{SO}_{3} \mathrm{TCP}$ & $\mathrm{H}$ & 47 & 21 \\
\hline
\end{tabular}

${ }_{55}{ }^{a}$ Determined by integration of ${ }^{1} \mathrm{H}$ NMR relative to pentachlorobenzene as an internal standard.

The aldehyde tolerance of the optimised conditions for the hydroacylation of PFPVS was next explored. A broad range of aldehydes was selected to be appraised under the reaction ${ }_{60}$ conditions, aldehydes 1b-p. A mixture of aliphatic and aromatic aldehydes was chosen. The appraisal of aliphatic aldehydes, in particular, was to set the work against a background of aldehydes that give rise to aldol-derived products under harsher ionic-based conditions for hydroacylation. The motivation to examine 65 aromatic aldehydes was due to them being poorly tolerated under previously reported conditions for hydroacylation of PFPVS. ${ }^{8 \mathrm{f}}$ 
Table 3 Hydroacylation of vinyl sulfonate $\mathbf{2}$ with aldehydes $\mathbf{1 b}$-p.

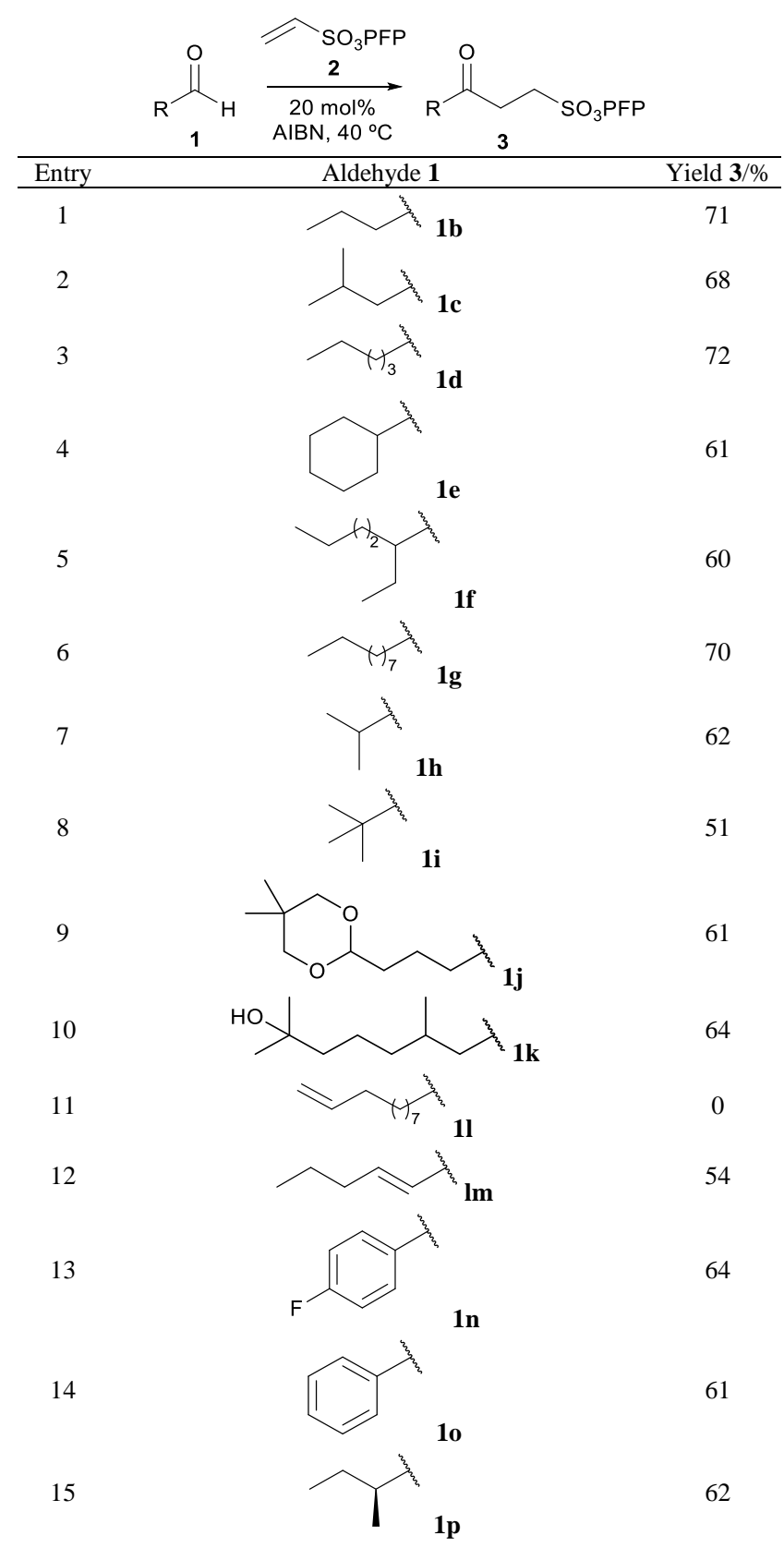

Conditions: Aldehyde 1 (1 mmol), vinyl sulfonate $2(1.5 \mathrm{mmol})$ and AIBN $(20 \mathrm{~mol} \%)$ in benzene (solvent) under argon at $40{ }^{\circ} \mathrm{C}$.

5 Pleasingly, good yields of ketone were generally observed across the broad selection of aldehydes trialled (Table 3). A variety of linear, $\alpha$-substituted and $\alpha, \alpha$-disubstituted aldehydes underwent hydroacylation in relatively good yields (Table 3, Entries 1-8). Moreover, the yield observed for isobutyraldehyde ${ }_{10} \mathbf{~ h}$ was in fact superior to that observed when using previously reported conditions that use a two-fold excess of aldehyde (Table 3, Entry 7). ${ }^{8 f}$ Functionalised aldehydes $\mathbf{1 j}$ and $\mathbf{1 k}$ were also well tolerated under the reaction conditions (Table 3, Entries 9-10). As expected, under free radical reaction conditions, application of

15 10-undecenal $\mathbf{1 l}$ resulted in polymerisation and no ketone product was isolated from the reaction mixture (Table 3, Entry 11).
Interestingly, however, an internal alkene conjugated with a carbonyl group was better tolerated and afforded ketone in respectable yield (Table 3, Entry 12). Under the mild reaction 20 conditions, minimal to zero aldol-derived product(s) were observed for any of the aliphatic aldehydes. Most pleasingly, use of aromatic aldehydes $\mathbf{1 n}$ and $\mathbf{1 0}$ also gave the respective ketones in good yield (Table 3, Entries 13-14). This is in sharp contrast to what was observed under previously reported reaction conditions, 25 i.e. $<10 \%$ yield. ${ }^{8 f}$ Finally, an aldehyde with an optically enriched $\alpha$-stereocentre, $(S)$-2-methylbutanal, was trialled. Reaction proceeded with retention of stereochemistry and highlights another clear advantage over common ionic methods which are more likely to racemise the stereocentre.

30 In conclusion, a methodology that allows for a shift from functionalising alkenes to functionalising aldehydes has been presented. This shift in focus has been enabled by the use of PFPVS, owing to its unique properties. The developed protocol has been shown to work across a series of aliphatic, 35 functionalised and aromatic aldehydes in good yields. Moreover, in certain cases, superior yields compared to those obtained in procedures where an excess of aldehyde was employed were observed. The tolerance of an $\alpha$-enantioenriched aldehyde highlights another advantage of using a mild radical-based 40 protocol. Owing to their established reactivity profile, ${ }^{8 b, f}$ the formed $\gamma$-keto-PFP-sulfonates will have application in a variety of fields.

\section{Acknowledgements}

The author gratefully acknowledges UCL, Dr Richard ${ }_{45}$ Fitzmaurice and Professor Stephen Caddick for useful discussions.

\section{Notes and references}

Department of Chemistry, University College London, 20 Gordon Street, London, WC1H OAJ, UK. Tel: +44 (0)20 7679 2077; E-mail:

50 v.chudasama@ucl.ac.uk

$\dagger$ Electronic Supplementary Information (ESI) available: $\left[{ }^{1} \mathrm{H}\right.$ and ${ }^{13} \mathrm{C}$ NMR spectra for all compounds]. See DOI: 10.1039/b000000x/

1 a) W. B. Motherwell and D. Crich, Free Radical Chain Reactions in Organic Synthesis, Academic Press, London, 1992; b) G. J.

55 Rowlands, Tetrahedron, 2009, 65, 8603-8655; c) G. J. Rowlands, Tetrahedron, 2010, 66, 1593-1636.

2 S. M. Kharasch, W. H. Urry and B. M. Kuderna, J. Org. Chem., 1949, 14, 248-253.

3 a) T. M. Patrick, J. Org. Chem., 1952, 17, 1009-1016; b) R. L. 60 Huang, J. Chem. Soc., 1956, 1749-1755.

4 a) L. M. van der Linde and A. J. A. van der Weerdt, Tetrahedron Lett., 1984, 25, 1201-1204; b) H. Stockman, J. Org. Chem., 1964, 29, 245; c) R. Dowbenko, J. Am. Chem. Soc., 1964, 86, 946-947; d) L. Friedman, J. Am. Chem. Soc., 1964, 86, 1885-1886; e) J. D. LaZerte

65 and R. J. Koshar, J. Am. Chem. Soc., 1955, 77, 910-914; f) H. Muramatsu and K. Inukai, J. Org. Chem., 1962, 27, 1572-1574; g) C. Chatgilialoglu, D. Crich, M. Komatsu and I. Ryu, Chem. Rev., 1999, 99, 1991-2069.

5 a) H. S. Dang, and B. P. Roberts, J. Chem. Soc. Perkin Trans. 1, 1998, 67-75; b) S. Tsujimoto, T. Iwahama, S. Sakaguchi, and Y. Ishii, Chem. Commun., 2001, 2352-2353; c) S. Tsujimoto, S. Sakaguchi, and Y. Ishii, Tetrahedron Lett., 2003, 44, 5601-5604; d) L. Melone and C. Punta, Beilstein J. Org. Chem., 2013, 9, 12961310.

756 a) J. C. Leung and M. J. Krische, Chem. Sci., 2012, 3, 2202-2209 and references therein; b) M. C. Willis, Chem. Rev., 2010, 110, 725-748 
and references therein; c) M. C. Willis, Reference Module in Chemistry, Molecular Sciences and Chemical Engineering, ed. G. A. Molander and P. Knochel, Elsevier, Oxford, $2^{\text {nd }}$ edn., 2014, vol. 4, ch. 4.17, pp. 961-994 and references therein.

57 a) F. Liu, X. Bugaut, M. Scheler, R. Fröhlich and F. Glorius, Angew. Chem. Int. Ed., 2011, 50, 12626-12630; b) X. Bugaut, F. Liu and F. Glorius, J. Am. Chem. Soc., 2011, 133, 8130-8133; c) A. T. Biju and F. Glorius, Angew. Chem. Int. Ed., 2010, 49, 9761-9764.

8 a) R. J. Fitzmaurice, J. M. Ahern, and S. Caddick, Org. Biomol. 10 Chem., 2009, 7, 235-237; b) V. Chudasama, R. J. Fitzmaurice, J. M. Ahern and S. Caddick, Chem. Commun., 2010, 46, 133-135; c) V. Chudasama, R. J. Fitzmaurice and S. Caddick, Nat. Chem., 2010, 2, 592-596; d) V. Chudasama, J. M. Ahern, R. J. Fitzmaurice and S. Caddick, Tetrahedron Lett., 2011, 52, 1067-1069; e) V. Chudasama, 15 R. J. Fitzmaurice, D. V. Dhokia, J. M. Ahern and S. Caddick, Chem. Commun., 2011, 47, 3269-3271; f) V. Chudasama, A. R. Akhbar, K. A. Bahou, R. J. Fitzmaurice and S. Caddick, Org. Biomol. Chem., 2013, 11, 7301-7317. 